\title{
Factor analysis identifies three separate symptom clusters in idiopathic pulmonary fibrosis
}

\author{
Severi Seppälä $\mathbb{1}^{1,2}$, Kaisa Rajala ${ }^{1,3}$, Juho Tuomas Lehto ${ }^{4}$, Eva Sutinen ${ }^{1,5}$, \\ Laura Mäkitalo ${ }^{1,5}$, Hannu Kautiainen ${ }^{6,7}$, Hannu Kankaanranta (10,9, \\ Mari Ainola ${ }^{1,5}$, Tiina Saarto ${ }^{10}$ and Marjukka Myllärniemi ${ }^{1,5}$
}

Affiliations: ${ }^{1}$ INDIVIDRUG Research Program, Research Programs Unit, Faculty of Medicine, University of Helsinki, Helsinki, Finland. ${ }^{2}$ Päijät-Häme Central Hospital, Dept of Respiratory Medicine, Lahti, Finland. ${ }^{3}$ Joint Municipal Authority for Health Care and Social Services in Keski-Uusimaa, Uusimaa, Finland. ${ }^{4}$ Palliative Care Centre and Dept of Oncology, Tampere University Hospital and Faculty of Medicine and Health Technology, University of Tampere, Tampere, Finland. ${ }^{5}$ Helsinki University Hospital, Heart and Lung Center, Helsinki, Finland. ${ }^{6}$ Primary Health Care Unit, Kuopio University Hospital, Finland. ${ }^{7}$ Folkhälsan Research Center, Helsinki, Finland. ${ }^{8}$ Faculty of Medicine and Health Technology, University of Tampere, Tampere, Finland. ${ }^{9}$ Dept of Respiratory Medicine, Seinäjoki Central Hospital, Seinäjoki, Finland. ${ }^{10}$ Helsinki University Hospital, Comprehensive Cancer Center, Dept of Palliative Care and Faculty of Medicine, University of Helsinki, Helsinki, Finland.

Correspondence: Mari Ainola, Lung factor, Research program unit INDIVIDRUG, University of Helsinki, Haartmaninkatu 3, 00290 Helsinki, Finland. E-mail: mari.ainolaahelsinki.fi

\section{ABSTRACT}

Background: Idiopathic pulmonary fibrosis (IPF) is a severe and progressive lung disease with a poor prognosis. Patients with IPF suffer from a high symptom burden, which impairs their health-related quality of life (HRQoL). Lack of research on IPF symptoms and their clustering, however, makes symptom-centred care challenging.

Methods: We sent two questionnaires, RAND 36-Item Health Survey and Edmonton Symptom Assessment System, to 300 patients from the FinnishIPF registry. Of the 300 patients, 245 (82\%) responded. We performed an exploratory factor analysis on the results to search for potential clustering of symptoms into factors.

Results: We found three distinct symptom factors: the emotional factor (including depression, anxiety, insomnia, loss of appetite and nausea), the pain factor (pain at rest or in movement) and the respiratory symptoms factor (shortness of breath, cough, tiredness and loss of wellbeing). Correlation was strong within the factors $\left(\rho_{\tau} 0.78-0.85\right)$ and also evident between them. The factors correlated with the different dimensions of HRQoL: the emotional factor with mental health (correlation coefficient $=-0.69)$ and vitality $(-0.63)$, the pain factor with bodily pain $(-0.72)$ and the respiratory symptoms factor with vitality $(-0.69)$, general health $(-0.64)$ and physical functioning $(-0.62)$.

Conclusion: We found three distinct symptom factors in IPF, of which respiratory and emotional factors showed the strongest association with decreasing HRQoL. Routine assessment of IPF patients' respiratory symptoms, mental health and pain are important as these may be linked with other symptoms and significantly impair the patient's HRQoL.

@ERSpublications

There are three distinct symptom factors (respiratory, emotional and pain) in IPF patients. The symptom factors are associated with impairment in different domains of quality of life, with the respiratory and emotional factors having a widespread impact. https://bit.ly/3inDezm

Cite this article as: Seppälä S, Rajala K, Lehto JT, et al. Factor analysis identifies three separate symptom clusters in idiopathic pulmonary fibrosis. ERJ Open Res 2020; 6: 00347-2020 [https:// doi.org/10.1183/23120541.00347-2020].

This article has supplementary material available from openres.ersjournals.com

Received: 4 June 2020 | Accepted after revision: 26 June 2020

Copyright $\odot$ ERS 2020. This article is open access and distributed under the terms of the Creative Commons Attribution Non-Commercial Licence 4.0 


\section{Introduction}

Idiopathic pulmonary fibrosis (IPF) is a progressive lung disease that impairs the health-related quality of life (HRQoL) of patients. Prognosis is poor, as only one in four patients survive 5 years after the diagnosis [1-5]. A high symptom burden, together with the varying progression rate of the disease, calls for early integrated palliative care $[1,6,7]$. However, the targeting and timing of symptom-centred care is difficult, because there is only a limited number of studies on symptoms and their impact on HRQoL in IPF patients.

The most common symptoms of IPF are shortness of breath and cough [8], which may start months or even years before the diagnosis $[9,10]$. Tiredness, anxiety, depression and pain are also reported by IPF patients [5]. An Australian study on IPF patients showed that dyspnoea, cough and depression had a high impact on HRQoL of patients [3], while moderate to severe dyspnoea correlated with a diminished HRQoL in all domains also in a Finnish study on IPF [5]. Although there are studies on individual symptoms of IPF patients, information on their concomitant occurrence and overall impact on HRQoL is needed to guide comprehensive symptom-centred care.

Studying clustering of symptoms may lead to the discovery of interrelated symptoms, and thereby help clinicians to understand the spectrum and interconnectedness of the symptoms associated with a particular disease. In factor analysis, a factor is a group or a cluster of known variables associated with a latent variable. By extension, we defined a symptom factor as a cluster of symptoms connected by an underlying variable. Clustering of symptoms has been studied in advanced cancer [11, 12], COPD [13], asthma [14], sleep apnoea [15] and lung cancer [16, 17]. To the best of our knowledge, despite a high symptom burden in IPF, no studies on symptom clustering in IPF have been published.

To understand the overall symptom burden in IPF, we explored whether identifiable symptom factors exist in a well-documented real-life cohort of IPF patients, and whether these factors have an impact on HRQoL.

\section{Methods}

\section{FinnishIPF registry study}

FinnishIPF, initiated in 2012, is a clinical prospective registry study of Finnish IPF patients. Virtually all IPF patients in Finland are at first evaluated in public central or university hospitals. The FinnishIPF registry includes all IPF patients, who have given informed consent to take part in the study, from these specialist centres. The diagnosis of IPF is made in accordance with the European Respiratory Society/ American Thoracic Society 2011/2015/2018 criteria [18-20]. A detailed description of the FinnishIPF study has been presented by KAUNISTO et al. [21].

\section{Patients}

300 IPF patients included in the FinnishIPF registry study were approached by mail in April 2015 and asked for a written informed consent to participate in our substudy on HRQoL in IPF [5]. Of these 300 patients, 42 refused to participate, 6 turned out not to have IPF, 3 died before answering the first questionnaire, 2 had received lung transplantation and 2 did not complete the Edmonton Symptom Assessment System (ESAS) questionnaire; thus, a total of 245 patients formed the final study population.

We used several different questionnaires to assess HRQoL in our study population $[5,22]$. For this paper, we analysed the results of the modified ESAS [23] and the RAND 36-Item Health Survey (RAND-36) [24].

\section{Questionnaires}

The ESAS symptom-based questionnaire measures pain, nausea, tiredness, insomnia, shortness of breath, wellbeing, depression, anxiety and loss of appetite on a numeric rating scale (NRS) from 0 (no symptoms) to 10 (the worst possible symptoms) [23]. The ESAS questionnaire allows the inclusion of additional symptoms. Thus, we added cough to the questionnaire and divided pain into pain at rest and pain in movement. Originally developed for cancer patients, ESAS is also frequently used to assess other patients in palliative care [23]. In ESAS, a symptom score $\geqslant 4$ on the NRS scale is commonly considered to represent a symptom with a moderate to severe intensity [23]. We have earlier found a number of patients reporting chest pain in IPF [5]. As a subanalysis, we examined the symptom of chest pain reported in ESAS more closely, in relation to coronary heart disease. We looked at patients treated in Helsinki University central area, as we had access to relevant medical records. Of the 43 patients treated in Helsinki University Central Hospital, 29 had reported chest pain in the ESAS questionnaire. We reviewed their university hospital and the medical records for primary and secondary healthcare.

RAND-36 is a frequently used, generic HRQoL questionnaire, with 36 items across eight domains: physical function, mental health, general health, social, mental and physical role functioning, bodily pain and vitality [24]. It is similar to the 36-Item Short Form Survey (SF-36) [25]. The two vary only in general health and pain scoring [24]. SF-36 is most widely used and has been previously validated and often used 
in IPF patients; however, we chose RAND-36, because it has reference values for the Finnish general population and is intended for use as a generic measure of HRQoL. A shortened version of the SF-36, known as SF-6D, is a single index health measure mostly utilised by health economists to calculate quality adjusted life years (QALY) $[25,26]$. In comparison to the eight dimensions of SF-36, SF-6D has no separate general health dimension and role limitations due to emotional problems have been merged with role limitations due to physical problems, resulting in a six-dimensional index [26, 27].

\section{Statistical analysis}

Data are presented as means with SD or as counts (n) with percentages (\%).

An exploratory factor analysis with iterated principal-factor method for factoring and promax-rotated factor loadings on correlation matrix was performed to identify related symptoms of ESAS. The strategies used to extract the number of factors were: the Kaiser criteria, which determines that components with eigenvalues lower than one should be excluded and the screen test of Cattell criteria. Internal consistency was estimated by calculating Cronbach's $\alpha$ internal consistency with bias corrected bootstrap 95\% confidence intervals. Adjusted correlation (partial) coefficients symptoms factors and RAND-36 domains were calculated by the Pearson method, using Sidak adjusted probabilities. According to Cohen [28, 29], the effect size is low if the value of $r$ varies around 0.10 , medium if $r$ varies around 0.30 , and large if $r$ varies more than 0.50. Stata 15.1 (StataCorp LP; College Station, Texas, USA) statistical package was used for the analysis. In assessing relation of chest pain coronary heart disease, an independent samples t-test was used to compare scale values and a Chi-squared test and Fisher's Exact test were used for comparison of categorical variables between different patient groups. A two-sided p-value $<0.05$ was accepted as statistically significant.

\section{Ethical statement}

This substudy is a part of the FinnishIPF study, which has a nationwide research permit in Finland from The National Institute for Health and Welfare (Dnro THL/1161/5.05.01/2012). In addition, approved positive statement was received from the ethics committee of the Hospital District of Helsinki and Uusimaa (HUS) (381/13/03/01/2014). All participants gave written informed consent.

\section{Results}

Patient characteristics are shown in table 1. Two-thirds of the patients were men and the mean time from the diagnosis of IPF was 3.4 years (SD 2.4). Comorbidities were found in $80 \%$ of the patients, of which cardiovascular diseases were the most common. Almost half (47\%) of the patients were never-smokers.

\section{Symptom factors}

Table 2 presents three factors found in the exploratory factor analysis and the symptoms belonging to them. The emotional factor included depression, anxiety, insomnia, nausea and loss of appetite; the pain factor included pain at rest or in movement; and the respiratory symptoms factor included shortness of breath, cough, tiredness and loss of wellbeing.

Cronbach's $\alpha$ for the emotional factor was 0.85 (95\% CI: $0.82-0.89$ ), for the pain factor 0.78 (95\% CI 0.71-0.85) and for the respiratory symptoms factor 0.78 (95\% CI $0.73-0.83$ ). There was also some correlation between the factors: the correlation coefficient between the emotional and the pain factor was 0.57 (95\% CI $0.47-0.64$ ), the emotional and the respiratory symptoms factor 0.58 (95\% CI $0.49-0.66$ ) and the pain and the respiratory symptoms factor 0.53 (95\% CI $0.43-0.61$ ).

Severity of the symptoms (mean NRS scores) grouped to their factors are shown in figure 1. The respiratory symptoms factor had the highest grand mean NRS score.

\section{Symptom factors and health-related quality of life (RAND-36)}

Correlations between the symptom factors and the different domains of HRQoL measured by RAND-36 are presented in table 3. The emotional factor had the strongest correlation with mental health and vitality, the pain factor with bodily pain and the respiratory symptoms factor with vitality, general health and physical functioning.

Figure 2 shows the correlations of the symptom factors with SF-6D general health summary, and with the mental and physical health summaries of the SF-6D. The emotional factor had the strongest correlation with the mental health summary and the general SF-6D, the pain factor with physical health summary and the respiratory symptoms factor with the physical health summary and the general SF-6D. 


\begin{tabular}{|c|c|}
\hline Age years & $74(48-92)$ \\
\hline Men & $161(66 \%)$ \\
\hline Duration of IPF years & $3.4 \pm 2.4$ \\
\hline Education years & $10 \pm 4$ \\
\hline Working & $21(9 \%)$ \\
\hline \multicolumn{2}{|l|}{ Smoking status } \\
\hline Smoker & $24(10 \%)$ \\
\hline Ex-smoker & 105 (43\%) \\
\hline Never-smoker & $116(47 \%)$ \\
\hline$F^{\prime \prime} C^{\#} L$ & $3.0 \pm 0.9$ \\
\hline FVC $\#$ \% pred & $83 \pm 17$ \\
\hline \multicolumn{2}{|l|}{ Comorbidities } \\
\hline Hypertension & $101(41 \%)$ \\
\hline Coronary heart disease & $53(22 \%)$ \\
\hline Diabetes & $47(19 \%)$ \\
\hline Cardiac insufficiency & $44(18 \%)$ \\
\hline COPD & $41(17 \%)$ \\
\hline Cancer & $41(17 \%)$ \\
\hline Asthma & $24(10 \%)$ \\
\hline Others & $38(16 \%)$ \\
\hline No comorbidities & $48(20 \%)$ \\
\hline \multicolumn{2}{|l|}{ RAND-36 domains } \\
\hline Physical functioning & $47 \pm 29$ \\
\hline Physical role function & $31 \pm 39$ \\
\hline Bodily pain & $65 \pm 27$ \\
\hline General health & $39 \pm 18$ \\
\hline Vitality & $53 \pm 23$ \\
\hline Social role function & $73 \pm 27$ \\
\hline Emotional role function & $51 \pm 42$ \\
\hline Mental health & $72 \pm 20$ \\
\hline
\end{tabular}

Data are presented as mean (range), $\mathrm{n}(\%)$ or mean \pm SD. ${ }^{\#}$ : FVC was recorded the time of the diagnosis. IPF: idiopathic pulmonary fibrosis; FVC: forced vital capacity; RAND-36: RAND 36-Item Health Survey.

\section{Comorbidities}

There was only a modest correlation between pain in movement, tiredness, shortness of breath and cough to coronary heart disease when correlation between the different symptoms and specific comorbidities adjusted for age and sex was analysed (supplementary table S1). No other significant correlations were found between these groups.

In our subanalysis of patients in Helsinki University area, 29 reported chest pain in the ESAS questionnaire. Six of them had a previous diagnosis of coronary heart disease, five received a new diagnosis and five were

TABLE 2 Exploratory factor analysis with promax-rotated factor loadings of the personal value priorities items

\begin{tabular}{|c|c|c|c|}
\hline Symptoms (ESAS) & Emotional factor & Pain factor & Respiratory symptoms factor \\
\hline Pain at rest & & 0.83 & \\
\hline Pain in movement & & 0.77 & \\
\hline Tiredness & & & 0.68 \\
\hline Nausea & 0.70 & & \\
\hline Depression & 0.87 & & \\
\hline Anxiety & 0.84 & & \\
\hline Insomnia & 0.54 & & \\
\hline Loss of appetite & 0.67 & & \\
\hline Shortness of breath & & & 0.74 \\
\hline Cough & & & 0.71 \\
\hline Wellbeing & & & 0.65 \\
\hline
\end{tabular}

Coefficients with values $<0.50$ not shown. The higher the coefficient, the stronger the correlation is. ESAS: Edmonton Symptom Assessment System. 
Emotional factor:

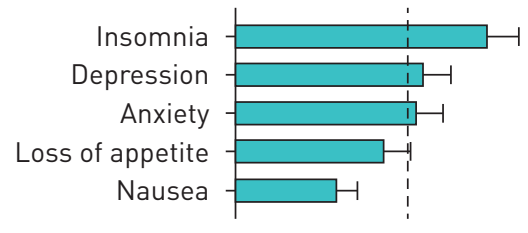

Pain factor:

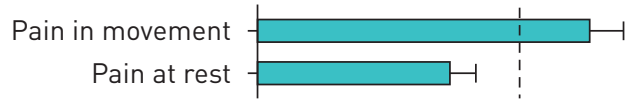

Respiratory symptoms factor:

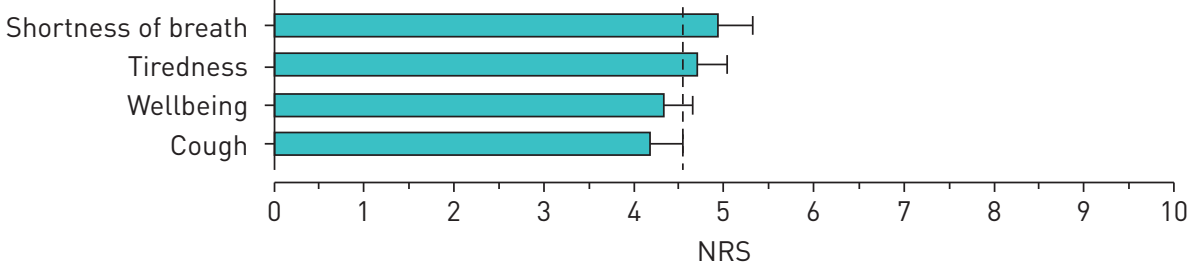

FIGURE 1 Mean Numeric Rating Scale (NRS) values for each of the Edmonton Symptom Assessment System (ESAS) symptoms, grouped according to their factors. The dashed lines indicate the grand means of symptoms in each of the factors.

examined for coronary heart disease but did not receive diagnosis. Thirteen patients were not examined (supplementary table S2). Four of the patients with previous coronary heart disease required new intervention, for two there was no obvious reason for the chest pain. For newly diagnosed coronary heart disease, this seemed the most relevant issue behind the chest pain. No correlation between reported pain and coronary heart disease could be found. Patients without coronary heart disease that mentioned chest pain in medical records $(n=11)$ had various reasons for the pain. These included degenerative findings in the thoracic spine/ribcage $(n=1)$, pneumonia $(n=1)$, pericarditis $(n=1)$ and aortic stenosis with atrial flutter $(n=1)$. For two patients the reason was not obvious but may have been related to gastrointestinal issues: one had chest pain when vomiting (possible gastroenteritis) and one after use of alcohol or a large meal. For the remaining $(n=5)$, there seemed to be no specific reason for the pain.

\section{Discussion}

In this cross-sectional study we found three symptom factors (emotional, pain and respiratory symptoms) in IPF patients. There was strong internal correlation and moderate correlation between the factors. The factors correlated with different domains of HRQoL in RAND-36: the emotional factor had the strongest correlation with mental health and vitality, the pain factor with bodily pain and the respiratory symptoms factor with vitality, general health and physical functioning.

Shortness of breath and cough are the most common symptoms of IPF and they impair HRQoL in IPF patients $[3,5]$. In our study, dyspnoea and cough were linked to tiredness and loss of wellbeing. These

TABLE 3 Correlation between the three symptom factors and the eight domains of RAND-36, adjusted for age and sex

\begin{tabular}{lccc} 
RAND-36 domains & Emotional factor & Pain factor & Respiratory symptoms factor \\
\hline Physical functioning & -0.39 & -0.40 & -0.62 \\
General health & -0.49 & -0.39 & -0.64 \\
Vitality & -0.63 & -0.42 & -0.69 \\
Mental health & -0.69 & -0.36 & -0.53 \\
Physical role function & -0.29 & -0.31 & -0.51 \\
Emotional role function & -0.39 & -0.27 & -0.38 \\
Social role function & -0.55 & -0.36 & -0.57 \\
Bodily pain & -0.48 & -0.72 & -0.46 \\
\hline
\end{tabular}

All correlations are significant (Sidak adjusted probabilities $p<0.001$ ). The lower the coefficient, the stronger the correlation is. RAND-36: RAND 36-Item Health Survey. 

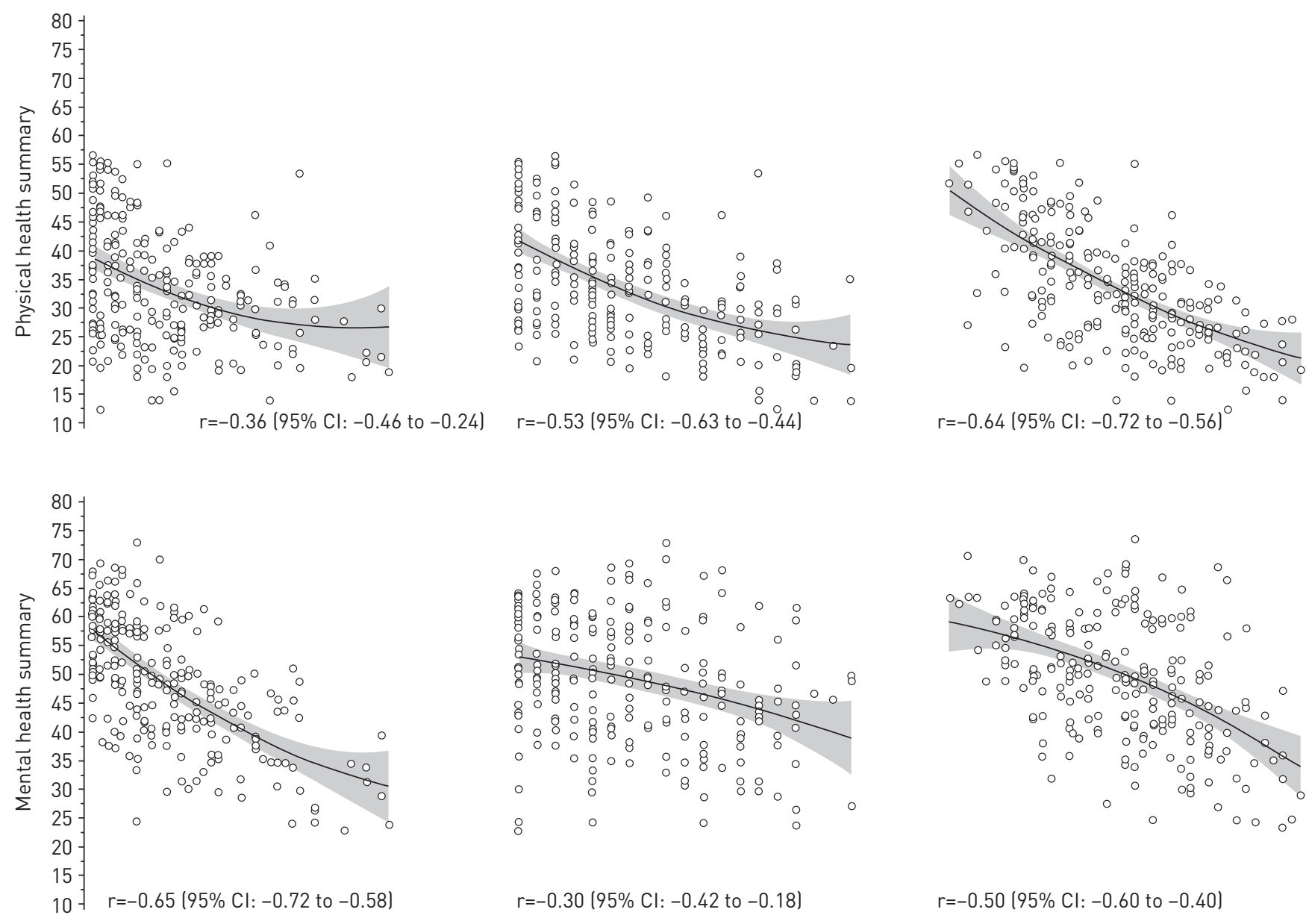

$r=-0.50(95 \% \mathrm{Cl}:-0.60$ to -0.40$)$
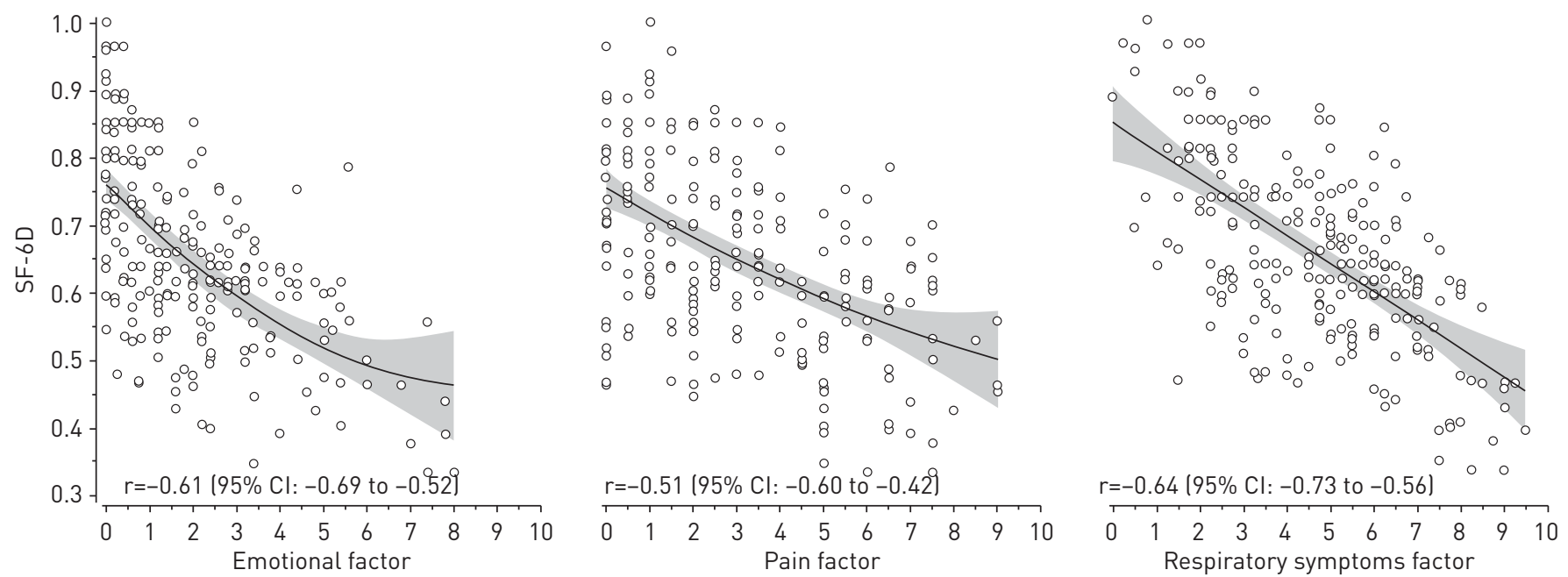

FIGURE 2 SF-6D physical health summary, mental health summary and general SF-6D compared to the three factors lemotional factor, pain factor and respiratory symptoms factor).

four symptoms formed the respiratory symptoms factor, which showed the highest severity of symptoms. The respiratory symptoms factor correlated with three domains of HRQoL: general health, physical functioning and vitality (i.e. having strength and energy), showing the widespread impact of dyspnoea on IPF patients' wellbeing. Increasing respiratory symptoms impair physical functioning and exercise capacity, which in turn decreases vitality and further impairs physical activity. This vicious circle of dyspnoea can over time cause patients' general health to decline, as can be seen in patients with lung cancer [30]. 
Depression and anxiety were linked with insomnia, loss of appetite and nausea in our study. Insomnia and loss of appetite can present as symptoms of depression [31] and nausea could be linked to both anxiety and depression [32]. As expected, the emotional factor showed a strong correlation with mental health. This is in line with previous studies, which show that depression and anxiety impair HRQoL in IPF patients $[33,34]$. However, the emotional factor also correlated with vitality in our study. Vitality not only reflects physical tiredness, but also mental exhaustion, which may explain the correlation between vitality and the emotional factor.

In contrast to the emotional and respiratory symptoms factors, pain factor had a rather specific correlation to bodily pain in RAND-36. In our study, pain formed a factor of its own, distinct from other symptoms. Pain, especially in movement and located on the chest, has previously been reported in IPF patients [5]. The aetiology of pain in IPF patients remains somewhat unclear, but because pain is not associated with the respiratory symptoms factor, it may have a mixed aetiology (e.g. musculoskeletal pain or some other type of pain not directly associated with IPF) or associated with other comorbidities or ageing.

Previous research on IPF symptoms is limited, and as far as we know, this is the first study to present symptom factors in IPF. However, there are studies on clustering of symptoms in patients with other lung diseases and in advanced cancer. HeNOCH et al. [16] studied the symptoms of 400 inoperable lung cancer patients and were able to identify three clusters of symptoms: pain (bowel problems, nausea, pain, loss of appetite and fatigue), respiratory (coughing and dyspnoea), and mood (depression, insomnia, worry and loss of concentration). In another study of 178 patients with advanced lung cancer and gefitinib treatment, nausea, lack of appetite, insomnia as well as pain were in the cluster of treatment associated symptoms instead of psychological or lung cancer symptom cluster [17]. The clustering of symptoms in the present IPF study differed from these lung cancer studies, as in IPF patients impaired wellbeing and tiredness were associated with respiratory symptoms, whereas nausea and loss of appetite were linked to emotional wellbeing in IPF patients. This can be, at least partly, explained by the different symptom burden in IPF and lung cancer. In IPF, respiratory symptoms are dominant, reflecting disease progression and impaired overall wellbeing. In inoperable lung cancer, in addition to respiratory symptoms, pain is one of the most significant concerns. Opioids are widely used in alleviation of cancer pain and as constipation, nausea and drowsiness are common side effects of opioids, their association with pain is understandable.

CHEUNG et al. [11] studied 1366 patients with advanced cancer and found two clusters of symptoms: one including fatigue, nausea, drowsiness, decreased appetite and dyspnoea and another one with depression and anxiety. Pain had a low factor loading $(<0.60)$ in both of the clusters, meaning it was not significantly linked to either of them. In another study by CHEUNG et al. [12], the clustering of symptoms differed depending on age ( $\leqslant 60$ and $>60$ years) and sex. Fatigue, drowsiness and wellbeing were not significantly associated with either of the clusters in patients over 60 years, but in patients $\leqslant 60$ years they clustered together with pain, depression and lack of appetite. Interestingly, fatigue, depression and anxiety changed clusters according to sex, and in men, nausea, drowsiness and wellbeing did not belong to either of the clusters. In our study every symptom belonged to a single factor. However, we did not look for symptom factors according to age and sex due to the size and the age distribution of our study population.

In a relatively small study of 130 COPD patients, LIM et al. [13] found three clusters of symptoms, two of which were in line with our factors. Their respiratory-functional cluster with dyspnoea, physical functioning and dry mouth resembled our respiratory symptoms factor, and their mood cluster with depression and anxiety was similar to our emotional factor. In contrast to our study, sleep problems, which in the present study were related to the emotional factor and fatigue, which in the present study was presented by tiredness and wellbeing of the respiratory symptoms factor, formed their own cluster. Contrary to our study, that particular study found no separate pain cluster.

It seems, that the number and composition of symptom clusters varies between different studies, possibly due to the heterogeneity of the study populations. Symptom factors in lung cancer, COPD and IPF were, however, comparable to one another $[13,16,17]$. Respiratory and mental symptom factors seem to be dominant, which is probably due to the major impact of respiratory symptoms in lung diseases and the emotional burden of a severe life-limiting disease. However, there seems to be some variation in the association of fatigue and insomnia depending on the study, as both can be related to emotional distress, but also to general weakness caused by the physical symptom burden and disease progression.

The symptom factors had a significant correlation to the different domains of HRQoL in RAND-36 in our study. Our results are in line with the findings of CHOI et al. [17], who reported that depression and lung cancer symptoms cluster (which resembles our respiratory symptoms factor) correlate with a lower HRQoL. Lim et al. [13] also found that in COPD patients, a higher symptom score in a symptom cluster leads to a lower HRQoL. These two studies [13, 17], together with our study, highlight the significant 
effect that symptoms can have on HRQoL in patients with lung diseases. In the present study respiratory and emotional symptoms had the biggest impact on HRQoL.

\section{Study strengths and limitations}

To the best of our knowledge, this is the first study to present symptom factors in IPF.

Other strengths of our study include a relatively large unselected sample of IPF patients, and symptom evaluation in a real-life setting. However, the cross-sectional nature of our study did not allow us to study the stability or changes of the symptom factors over time. In addition, we were not able to compare our symptom factors to lung function tests or radiographic findings as these were not done at the same time with the questionnaires. It was also not possible to analyse the exact data of therapy, because the implementation of the patients' therapy has not been monitored. For this reason, we cannot say whether the use of oxygen and/or antifibrotic therapy could have had an impact on patients' symptoms and wellbeing.

\section{Conclusions}

We found three symptom factors in IPF patients (respiratory symptoms, emotional and pain factor). The respiratory symptoms factor showed the highest symptom burden and was associated with impaired overall wellbeing. Increasing symptom burden in respiratory and emotional factors showed the strongest association with decreasing HRQoL. In order to improve IPF patients' general wellbeing and HRQoL, optimal treatment of respiratory symptoms, rehabilitation and psychosocial support are vital, and routine symptom assessment should be a part of everyday clinical practice.

Conflict of interest: S.Seppälä reports a lecture fee from Roche Norge AS outside the submitted work. K. Rajala reports lecture fees from Boehringer Ingelheim, Roche and Kyowa Kirin; and grants from Väinö and Laina Kivi Foundation, the Finnish Anti-tuberculosis Society, and Helsinki University Cancer Center, all outside the submitted work. J.T. Lehto reports payments for lectures from Roche, Boehringer and Novartis, outside the submitted work. E. Sutinen has nothing to disclose. L. Mäkitalo has nothing to disclose. H. Kautiainen has nothing to disclose. H. Kankaanranta reports fees for lectures and consulting, costs for attending an international congress and a research grant to their institution from AstraZeneca; fees for consulting from Chiesi Pharma AB; fees for lectures and consulting, and costs for attending an international congress from Boehringer Ingelheim; fees for lectures and consulting from Novartis; fees for lectures from Mundipharma; fees for lectures and consulting, and cost for attending international congress from Orion Pharma; fees for consulting from SanofiGenzyme; and fees for lectures and consulting from GlaxoSmithKline, all outside the submitted work. M. Ainola has nothing to disclose. T. Saarto has nothing to disclose. M. Myllärniemi has nothing to disclose.

Support statement: S. Seppälä reports a grant from The Research Foundation of the Pulmonary Diseases HES, Päijät-Hämeen keuhkosairauksien tutkimussäätiö and SKLY Finnish Respiratory Society during the conduct of the study. The FinnishIPF study is funded by grants from the Sigrid Jusélius Foundation, the Helsinki University Hospital funds and the Nummela Sanatorium Foundation and Boehringer Ingelheim. Funding information for this article has been deposited with the Crossref Funder Registry.

\section{References}

1 Sköld CM, Bendstrup E, Myllärniemi M, et al. Treatment of idiopathic pulmonary fibrosis: a position paper from a Nordic expert group. J Intern Med 2017; 281: 149-166.

2 van Manen MJG, Geelhoed JJM, Tak NC, et al. Optimizing quality of life in patients with idiopathic pulmonary fibrosis. Ther Adv Respir Dis 2017; 11: 157-169.

3 Glaspole IN, Chapman SA, Cooper WA, et al. Health-related quality of life in idiopathic pulmonary fibrosis: data from the Australian IPF Registry. Respirology 2017; 22: 950-956.

4 Kreuter M, Swigris J, Pittrow D, et al. Health-related quality of life in patients with idiopathic pulmonary fibrosis in clinical practice: Insights-IPF registry. Respir Res 2017; 18: 139.

5 Rajala K, Lehto JT, Sutinen E, et al. mMRC dyspnoea scale indicates impaired quality of life and increased pain in patients with idiopathic pulmonary fibrosis. ERJ Open Res 2017; 3: 00084-2017.

6 Rajala K, Lehto JT, Saarinen M, et al. End-of-life care of patients with idiopathic pulmonary fibrosis. BMC Palliat Care 2016; 15: 1-6.

7 Raghu G, Richeldi L. Current approaches to the management of idiopathic pulmonary fibrosis. Respir Med 2017; 129: $24-30$.

8 Lee JS, McLaughlin S, Collard HR. Comprehensive care of the patient with idiopathic pulmonary fibrosis. Curr Opin Pulm Med 2011; 17: 348-354.

$9 \quad$ Lederer DJ, Martinez FJ. Idiopathic pulmonary fibrosis. N Engl J Med 2018; 378: 1811-1823.

10 Hewson T, McKeever TM, Gibson JE, et al. Timing of onset of symptoms in people with idiopathic pulmonary fibrosis. Thorax 2018; 73: 683-685.

11 Cheung WY, Le LW, Zimmermann C. Symptom clusters in patients with advanced cancers. Support Care Cancer 2009; 17: 1223-1230.

12 Cheung WY, Le LW, Gagliese L, et al. Age and gender differences in symptom intensity and symptom clusters among patients with metastatic cancer. Support Care Cancer 2011; 19: 417-423.

13 Lim KE, Kim SR, Kim HK, et al. Symptom clusters and quality of life in subjects with COPD. Respir Care 2017; 62: 1203-1211.

14 Ilmarinen P, Tuomisto LE, Niemelä O, et al. Cluster analysis on longitudinal data of patients with adult-onset asthma. J Allergy Clin Immunol Pract 2017; 5: 967-978. e3. 
15 Ye L, Pien GW, Ratcliffe SJ, et al. The different clinical faces of obstructive sleep apnoea: a cluster analysis. Eur Respir J 2014; 44: 1600-1607.

16 Henoch I, Lövgren M. The influence of symptom clusters and the most distressing concerns regarding quality of life among patients with inoperable lung cancer. Eur J Oncol Nurs 2014; 18: 236-241.

17 Choi S, Ryu E. Effects of symptom clusters and depression on the quality of life in patients with advanced lung cancer. Eur J Cancer Care (Engl) 2018; 27: 1-8.

18 Raghu G, Collard HR, Egan JJ, et al. An Official ATS/ERS/JRS/ALAT statement: idiopathic pulmonary fibrosis: evidence-based guidelines for diagnosis and management. Am J Respir Crit Care Med 2011; 183: 788-824.

19 Raghu G, Rochwerg B, Zhang Y, et al. An official ATS/ERS/JRS/ALAT clinical practice guideline: treatment of idiopathic pulmonary fibrosis. An update of the 2011 clinical practice guideline. Am J Respir Crit Care Med 2015 192: e3-e19.

20 Raghu G, Remy-Jardin M, Myers JL, et al. Diagnosis of idiopathic pulmonary fibrosis: an official ATS/ERS/JRS/ ALAT clinical practice guideline. Am J Respir Crit Care Med 2018; 198: e44-e68.

21 Kaunisto J, Kelloniemi K, Sutinen E, et al. Re-evaluation of diagnostic parameters is crucial for obtaining accurate data on idiopathic pulmonary fibrosis. BMC Pulm Med 2015; 15: 92.

22 Rajala K, Lehto JT, Sutinen E, et al. Marked deterioration in the quality of life of patients with idiopathic pulmonary fibrosis during the last two years of life. BMC Pulm Med 2018; 18: 172.

23 Hui D, Bruera E. The Edmonton symptom assessment system 25 years later: past, present, and future developments. J Pain Symptom Manage 2017; 53: 630-643.

24 Hays RD, Sherbourne CD, Mazel RM. The Rand 36-item health survey 1.0. Health Econ 1993; 2: 217-227.

25 Ware JE, Sherbourne CD. The MOS 36-item short-form health survey (SF-36). I. Conceptual framework and item selection. Med Care 1992; 30: 473-483.

26 Brazier J, Roberts J, Deverill M. The estimation of a preference-based measure of health from SF-36. J Health Econ 2002; 21: 271-292.

27 Brazier J, Roberts J, Tsuchiya A, et al. A comparison of the EQ-5D and SF-6D across seven patient groups. Health Econ 2004; 13: 873-884

28 Cohen J. Statistical power analysis for the behavioral sciences. Hillsdale, NJ, Lawrence Erlbaum Associates, 1988.

29 Cohen J. A power primer. Psych bull 1992; 112: 155-159.

30 Long NH, Thanasilp S, Thato R. A causal model for fatigue in lung cancer patients receiving chemotherapy. Eur J Oncol Nurs 2016; 21: 242-247.

31 Baxter LC. Appetite changes in depression. Am J Psychiatry 2016; 173: 317-318.

32 Haug TT, Mykletun A, Dahl AA. The prevalence of nausea in the community: psychological, social and somatic factors. Gen Hosp Psychiatry 2002; 24: 81-86.

33 Matsuda T, Taniguchi $\mathrm{H}$, Ando $\mathrm{M}$, et al. Depression is significantly associated with the health status in patients with idiopathic pulmonary fibrosis. Intern Med 2017; 56: 1637-1644.

34 Lee YJ, Choi SM, Lee YJ, et al. Clinical impact of depression and anxiety in patients with idiopathic pulmonary fibrosis. PLoS ONE 2017; 12: e0184300. 